\title{
Impact of general reionization scenarios on extracting inflationary parameters
}

\author{
Stefania Pandolfi \\ University of Rome La Sapienza \\ E-mail: stefaniapandolfi@gmail.com
}

\begin{abstract}
Determination of whether the Harrison-Zel'dovich spectrum for primordial scalar perturbations is consistent with observations is sensitive to assumptions about the reionization scenario. In light of this result, we revisit constraints on inflationary models using more general reionization scenarios. While the bounds on the tensor-to-scalar ratio are largely unmodified, when different reionization schemes are addressed, hybrid models are back into the inflationary game. In the general reionization picture, we reconstruct both the shape and amplitude of the inflaton potential. We discuss how relaxing the simple reionization restriction affects the reconstruction of the potential through the changes in the constraints on the spectral index, the tensor-to-scalar ratio and the running of the spectral index. We also find that the inclusion of other CMB data in addition to the WMAP data excludes the very flat potentials typical of models in which the inflationary evolution reaches a late-time attractor, as a consequence of the fact that the running of the spectral index is constrained to be different from zero at $99 \%$ confidence level.
\end{abstract}

25th Texas Symposium on Relativistic Astrophysics

December 6-10, 2010

Heidelberg, Germany 


\section{Introduction}

Inflation predicts $n \simeq 1$, but usually $n \neq 1$. A scale-invariant scalar power spectrum corresponding to the value $n=1$ is the model proposed by Harrison, Zel'dovich, and Peebles [1]. A value of the spectral index $n$ slightly different from unity would strongly point to the inflationary paradigm as the mechanism responsible for providing the initial conditions for structure formation. In addition, in many inflationary models the amplitude of gravitational waves is proportional to $|n-1|$. Confirmation of a deviation from a scale-invariant power spectrum would encourage the gravitational waves hunters to keep searching for the detection of a nonzero tensor amplitude ${ }^{1}$.

The most recent analysis by the Wilkinson Microwave Anisotropy Probe (WMAP) team of their seven-year data [3] rule out the Harrison-Zel'dovich $(\mathrm{H}-\mathrm{Z})$ primordial power spectrum at more than $3 \sigma$ when ignoring tensor modes: $n=0.968 \pm 0.012$. But this, as well as most other previously derived constraints from CMB data on cosmological parameters have assumed a "sudden" and complete reionization at a single redshift $z_{r}$. The electron ionization fraction $x_{e}(z)$ is such that for $z \ll z_{r} x_{e}(z)=1\left(x_{e}(z)=1.08\right.$ for $z<3$ in order to take into account Helium recombination) and $x_{e}(z)=2 \times 10^{-4}$ for $z>z_{r}$, i.e., joining the value after primordial recombination with a smooth interpolation.

The process of structure formation that led to gravitational collapse of objects in which the first stars formed are still subject to theoretical and observational uncertainties. As these first sources began to illuminate their local neighborhoods, the HI present in the IGM was "reionized." Since the precise details of reionization processes are currently unknown, it is mandatory to explore the imprints of general reionization histories on the CMB spectra. In a precursor study we demonstrated that in a general reionization scenario the Harrison-Zel'dovich spectrum $(n=1)$ is perfectly consistent with observations [6]. In this study we shall also include information from tensors modes, showing that inflationary models that are ruled out in the sudden reionization scheme are allowed in more general reionization scenarios. We also reconstruct both the shape and the amplitude of the inflationary potential $V(\phi)$ allowed by current data in both sudden and general reionization schemes. The relevant parameters to distinguish among inflationary models are $n$ and $r$ [8],[7]. The different classes of models are characterized by the relation between these two parameters, or equivalently, by the relation between the slow-roll parameters $\varepsilon$ and $\eta$. At lowest order in the slow-roll approximation we can divide the inflationary models into three general types: large-field, small-field and hybrid.

\section{ANALYSIS METHOD}

We adopt two different methods for parametrization of the reionization history. With the first method, developed in Ref. [5], the reionization history is parametrized as a free function of redshift by decomposing $x_{e}(z)$ into its principal components: $x_{e}(z)=x_{e}^{f}(z)+\sum_{\mu} m_{\mu} S_{\mu}(z)$, where the principal components, $S_{\mu}(z)$, are the eigenfunctions of the Fisher matrix that describes the dependence of the polarization spectra on the electron ionization fraction $x_{e}(z), m_{\mu}$ are the amplitudes of the principal components for a particular reionization history, and $x_{e}^{f}(z)$ is the WMAP fiducial

\footnotetext{
${ }^{1}$ For a discussion of slow-roll inflation models with $n=1$, see Ref. [2].
} 
model at which the Fisher matrix is computed and from which the principal components are obtained. In what follows we use the publicly available $S_{\mu}(z)$ functions and vary the amplitudes $m_{\mu}$ for $\mu=1, \ldots, 5$ for the first five eigenfunctions. Hereafter we refer to this method as the $\mathrm{MH}$ (Mortonson-Hu) case.

In a second approach we sample the evolution of the ionization fraction $x_{e}$ as a function of redshift $z$ at seven points $\left(z=9,12,15,18,21,24\right.$, and 27), and interpolating the value of $x_{e}(z)$ between them with a cubic spline. For $30<z$ we fix $x_{e}=2 \times 10^{-4}$ as the value of $x_{e}$ expected before reionization (and after primordial recombination), while $x_{e}=1$ for $3<z<6$ and $x_{e}=1.08$ for $z<3$ in order to be in agreement with both Helium ionization and Gunn-Peterson test observations. This approach is very similar to the one used in Ref. [9], and we will refer to it as the LWB (LewisWeller-Battye) case. We then modified the Boltzmann CAMB code [10], incorporating the two generalized reionization scenarios and extracted cosmological parameters from current data using a Monte Carlo Markov Chain (MCMC) analysis based on the publicly available MCMC package cosmomc [11]. We consider here a flat $\Lambda \mathrm{CDM}$ universe described by a set of cosmological parameters $\left\{\omega_{b}, \omega_{c}, \Theta_{s}, n, \log \left[10^{10} A_{s}\right], r, n_{\text {run }}\right\}$, where $\omega_{b} \equiv \Omega_{b} h^{2}$ and $\omega_{c} \equiv \Omega_{c} h^{2}$ are the physical baryon and cold dark matter densities relative to the critical density, $\Theta_{s}$ is the ratio between the sound horizon and the angular diameter distance at decoupling, $A_{s}$ is the amplitude of the primordial spectrum, $n$ is the scalar spectral index, $r$ is the tensor-to-scalar ratio, and $n_{r u n} \equiv d n / d \ln k$ is the running of the scalar spectral index. The extra parameters needed to describe reionization are the five amplitudes of the eigenfunctions for the MH case, or the seven amplitudes in the seven bins for the LWB case.

Our basic dataset is the seven-year WMAP data [3] (temperature and $E$ polarization) with the routine for computing the likelihood supplied by the WMAP team. We refer to this basic dataset as "WMAP7". We also consider an extended dataset, to which we refer as "CMB-ALL". This larger dataset includes the WMAP data, the CMB data from BOOMERanG [12], QUAD [13], ACBAR [14], and BICEP [15].

\section{RESULTS}

Table 1 summarizes the main results of the analysis for different cosmological data sets, showing the constraints on $n$ and $r$ for the MH, LWB, and sudden reionization schemes. When the sudden reionization assumption is relaxed, the mean values of $n$ and $r$ tend to shift to higher values. The shift in $n$ was already noted in the previous paper [6]. The importance of this shift is that in a general reionization scheme the $\mathrm{H}-\mathrm{Z}$ spectrum $(n=1)$ is perfectly consistent. In fact, in the framework of the minimal, six-parameter $\Lambda \mathrm{CDM}$ model (i.e. without tensor modes nor running of the spectral index), $n=1$ is ruled out at the $99 \%$ level [3]. However, as seen from the values reported in Table 1, the presence of tensors and possibly of a running spectral index in the analysis allows for a $\mathrm{H}-\mathrm{Z}$ spectrum even in the sudden reionization scheme, at $95 \%$ confidence level (c.l.). As a matter of fact, in the $\mathrm{MH}$ reionization case without running of the spectral index, the $\mathrm{H}-\mathrm{Z}$ spectrum is even inside the $68 \%$ c.l. for both the WMAP7 and CMB-ALL datasets. Moreover, in the case where both tensors and running are included, the mean value for $n$ is always found to be larger than one. This also happens for the MH reionization scenario without running, using the WMAP7 


\begin{tabular}{|c|c|c|c|c|c|c|}
\hline & \multicolumn{3}{|c|}{ WMAP7 } & \multicolumn{3}{|c|}{ CMB-ALL } \\
\hline & Sudden & MH & LWB & Sudden & MH & LWB \\
\hline \multicolumn{7}{|c|}{ without running of scalar spectral index } \\
\hline$n$ & $0.987 \pm 0.040$ & $1.010 \pm 0.054$ & $0.992 \pm 0.042$ & $0.974 \pm 0.032$ & $0.985 \pm 0.040$ & $0.977 \pm 0.034$ \\
\hline$r$ & $r \leq 0.373$ & $r \leq 0.376$ & $r \leq 0.371$ & $r \leq 0.251$ & $r \leq 0.266$ & $r \leq 0.275$ \\
\hline \multicolumn{7}{|c|}{ with running of scalar spectral index $95 \%$ c.l. } \\
\hline$n$ & $1.067 \pm 0.124$ & $1.080 \pm 0.129$ & - & $1.094 \pm 0.104$ & $1.106 \pm 0.108$ & - \\
\hline$r$ & $r \leq 0.497$ & $r \leq 0.515$ & - & $r \leq 0.450$ & $r \leq 0.445$ & - \\
\hline$\alpha$ & $-0.040 \pm 0.057$ & $-0.036 \pm 0.062$ & - & $-0.056 \pm 0.043$ & $-0.058 \pm 0.045$ & - \\
\hline \multicolumn{7}{|c|}{ with running of scalar spectral index $99 \%$ c.l. } \\
\hline$n$ & $0.913 \leq n \leq 1.221$ & $0.918 \leq n \leq 1.234$ & - & $0.968 \leq n \leq 1.216$ & $0.984 \leq n \leq 1.260$ & - \\
\hline$r$ & $r \leq 0.665$ & $r \leq 0.688$ & - & $r \leq 0.551$ & $r \leq 0.619$ & - \\
\hline$\alpha$ & $-0.098 \leq \alpha \leq 0.037$ & $-0.098 \leq \alpha \leq 0.050$ & - & $-0.099 \leq \alpha \leq-0.001$ & $-0.117 \leq \alpha \leq-0.001$ & - \\
\hline
\end{tabular}

Table 1: $95 \%$ and $99 \%$ c.l. constraints on $n, r$, and $\alpha \equiv n_{\text {run }}$ from the WMAP7 and CMB-ALL datasets, assuming different reionization scenarios.

dataset. In general, when additional data from other CMB probes are added to the WMAP7 data, the constraints on $n$ and $r$ are shifted back toward lower values.

For what concerns the tensor-to-scalar ratio $r$ and the running of the spectral index $n_{\text {run }}$, their $95 \%$ c.l. constraints barely change when the reionization history is modified, as expected, due to the large uncertainties on $r$ and $n_{\text {run }}$.

The shift induced on allowed values of inflationary parameters $n$ and $r$ by different assumptions for the reionization history is important for the subsequent constraints on inflationary models. To study this, we have reconstructed the relation between $n$ and $r$ for one choice of inflationary potentials for each class of models named in the previous section, and we have plotted these relations in the $n-r$ plane, together with the cosmological constraints. Following Ref. [7] we can easily develop the different expressions concerning the $n-r$ parameter space. It is also straightforward to relate $r$ with $N$ (the number of $e$-foldings before the end of inflation), which allows us to draw points with $N=50$ (squares) and $N=60$ (circles) in Fig. 1.

For hybrid models, the potential chosen is $V(\phi)=\Lambda^{4}\left[1-\alpha\left(m_{P l} / \phi\right)^{p}\right]$, based on potentials generated in dynamical SUSY breaking models [16]. In the sudden reionization scenario with negligible running of the spectral index these hybrid models are highly disfavored; in more general reionization schemes such models are allowed by WMAP7 data; see Fig. 1. When more CMB data sets are included in the analysis, hybrid inflation models with a blue tilt are again disfavored at 95\% c.l., even in the more general reionization scenarios considered here; see the CMB-ALL part of Fig. 1. When a running scalar spectral index is allowed, hybrid models are perfectly compatible with data, regardless of the assumptions about the reionization processes; see Fig. 1, lower panels.

The LWB reionization scheme leads to very similar constraints to those of $\mathrm{MH}$ parametrization on the $n-r$ plane (albeit slightly closer to the sudden reionization case).

We also forecast future constraints from the Planck experiment with the specifications of Ref. [17], assuming a fiducial model with $n=0.96, r=0.05$ and sudden reionization. Fitting the 

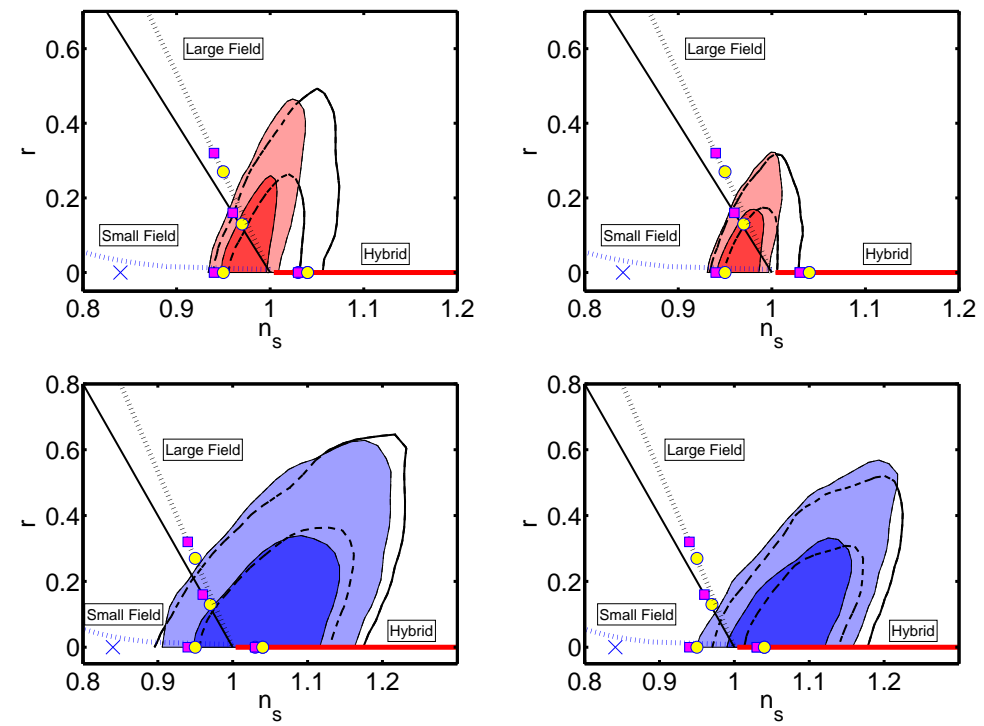

Figure 1: Two-dimensional contour plots at the $68 \%$ and $95 \%$ confidence levels without running (upper panels) and with running of the scalar spectral index (lower panels) of the scalar spectral index for the WMAP7 data (left figures) and the CMB-ALL data set (right figures). Shaded contours correspond to the sudden reionization approximation, while open contours model reionization as $\mathrm{MH}$. The dark solid (dashed) lines refer to large-field models with $p=2(p=4)$. The lighter cross (dashed curves) depict small-field models with $p=2(p=4)$. The solid horizontal line that basically coincides with the $x$ axis depicts hybrid models with $p=2$ (the $p=4$ case basically overlaps the $p=2$ case). The filled circles (squares) denote the points in the parameter space for which the number of $e$-folds $N$ is equal to 60 (50).

data assuming a more general reionization scenario (in this case $\mathrm{MH}$ reionization), we obtain: $n=0.960 \pm 0.008, r=0.0536 \pm 0.022$. Notice that Planck will be able to tell $n \neq 1$ at a very high confidence level even when fitting sudden reionization in the framework of a more general reionization scheme. Planck data will also be sensitive to the tensor-to-scalar ratio at the $95 \%$ c.l. for $r \geq 0.05$.

\section{Monte Carlo Reconstruction of the inflationary potential}

In this section we make use of the technique known as Monte Carlo reconstruction, a stochastic method for inverting observational constraints to obtain an ensemble of inflationary potentials compatible with observations. The method is described in more detail in Refs. [18, 19, 20] and references therein.

In order to calculate an ensemble of potentials that are compatible with observations, we proceed in the following way: choose random initial values for the inflationary parameters from a given ensamble of ranges, more and more restricted for higher and higher order of slow-roll parameters, truncated at $M=6$. Evolve forward in time $(d N<0)$ until either $(a)$ inflation ends $(\varepsilon>1)$, or (b) the evolution reaches a late-time fixed point $\left(\varepsilon=\lambda_{\mathrm{H}}^{(\ell)}=0, \sigma=\right.$ const). In case $(a)$, evolve $N$ $e$-folds backwards in time from the end of inflation and calculate the observables $n-1, r$, and the running $n_{\text {run }}$ at that time; in case $(b)$, calculate the observables at the time the evolution reaches the 
fixed point. Repeat the above procedure $N_{M C}$ times. Then choose a window of acceptable values for the observables $n-1, r$, and the running $n_{r u n}$, and then extract from the $N_{M C}$ models those that satisfy the observational constraints. Finally, reconstruct the potential for these models, following the procedure described above.

We have implemented the procedure described above with $N_{M C}=5 \times 10^{5}$. We consider four sets of observational constraints, conservatively corresponding to the $99 \%$ confidence regions obtained for the WMAP7 and CMB-ALL datasets in the two cases of sudden and MH reionization scenarios with running of the scalar spectral index. These are reported in Table 1. We show a sample of 300 reconstructed potentials in Fig. 2, distinguishing between models where the numerical integration reaches the end of inflation (red solid curves) and those where the evolution reaches a fixed point (blue dashed curves). We have rescaled all the potentials so that $V(\phi=0)=1$ and $0 \leq \phi \leq 1$, so that the figure only contains information concerning the shape of the inflationary potential. We see that the WMAP7 data alone do not really constrain the shape of the inflationary potential, even when more general models of reionization are considered. However, the situation changes dramatically when other CMB experiments are included, as it can be seen from the right panels of Fig. 2. In particular, the addition of these data constraints the inflation potential to be of the "inflation ends" kind. The very flat potentials typical of models in which the evolution reaches a fixed point are instead excluded (independently on the details of the reionization model). The reason is the following. The late-time attractor of the "fixed point" models produces very flat corresponding potentials: in terms of the observables, it means that in general these models predict $n_{\text {run }} \simeq 0$; we have explicitly checked that all the "fixed point" models generated in our MonteCarlo have $-0.8 \times 10^{-3} \leq n_{\text {run }} \leq 1.8 \times 10^{-3}$. However, when the larger dataset is considered, observations exclude models with $n_{\text {run }}>-10^{-3}$ at $99 \% \mathrm{CL}$, see Table 1 . Thus only potentials of the "inflation ends" kind, able in principle to produce "large" (in absolute value) runnings are allowed. We also notice that the inclusion of more general models of reionization does not appreciably change the constraints on the shape of the potential. Other than the shape of the potential, it is also important to constrain its amplitude. The reconstruction procedure described above does not yield the amplitude of the potential; this has to be fixed from some observational input, like the normalization of the Hubble parameter. We choose to normalize the Hubble parameter through the condition on the density contrast, $\delta \rho / \rho$. Once the normalization condition has been enforced, we find that in all the four cases, $V(\phi) \lesssim 10^{-11} \mathrm{~m}_{\mathrm{Pl}}^{4}$. This correspond to an upper limit to the energy scale of inflation of about $10^{16} \mathrm{GeV}$.

\section{References}

[1] E. R. Harrison, Phys. Rev. D 1, 2726 (1970); Y. B. Zel'dovich, Mon. Not. Roy. Astron. Soc. 160, (1972); P. J. E. Peebles and J. T. Yu Astrophys. J. 162, 815 (1970).

[2] A. Vallinotto, E. J. Copeland, E. W. Kolb, A. R. Liddle and D. A. Steer, Phys. Rev. D 69, 103519 (2004); A. A. Starobinsky, JETP Lett. 82, 169 (2005).

[3] E. Komatsu et al., arXiv:1001.4538 [astro-ph.CO]; D. Larson et al., arXiv:1001.4635 [astro-ph.CO].

[4] M. Zaldarriaga et al., arXiv:0811.3918 [astro-ph].

[5] M. J. Mortonson and W. Hu, Astrophys. J. 686, L53 (2008) [arXiv:0804.2631 [astro-ph]]. 

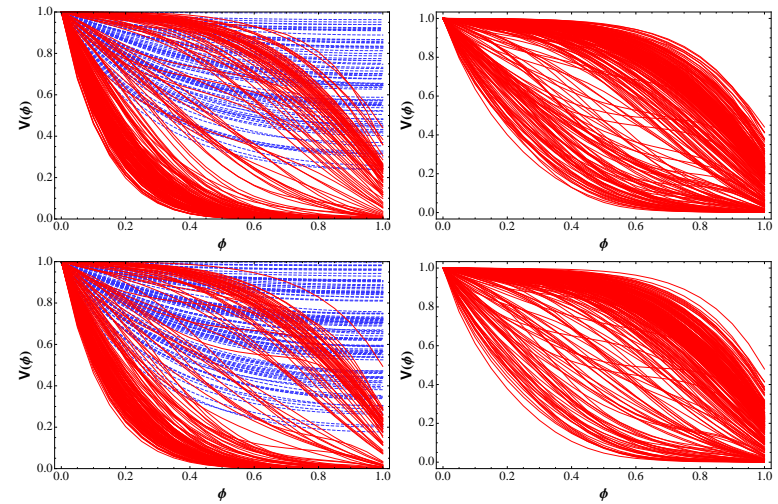

Figure 2: Sample of three hundred reconstructed potentials for the four sets of observational constraints discussed in the text. The potentials are calculated at the $99 \%$ c.l. The red solid (blue dashed) curves correspond to potentials of the "inflation ends" ("attractor") kind. All the potentials have been rescaled so that $V(\phi=0)=1$, and $0 \leq \phi \leq 1$. Upper left panel: WMAP7 with sudden reionization; upper right panel: CMB-ALL with sudden reionization; lower left panel: WMAP7 with MH reionization; lower right panel: CMB-ALL with MH reionization.

[6] S. Pandolfi et al., Phys. Rev. D 81, 123509 (2010) [arXiv:1003.4763 [astro-ph.CO]].

[7] W. H. Kinney, E. W. Kolb, A. Melchiorri and A. Riotto, Phys. Rev. D 74, 023502 (2006) [arXiv:astro-ph/0605338]; W. H. Kinney, E. W. Kolb, A. Melchiorri and A. Riotto, Phys. Rev. D 78, 087302 (2008) [arXiv:0805.2966 [astro-ph]].

[8] S. Dodelson, W. H. Kinney and E. W. Kolb, Phys. Rev. D 56, 3207 (1997) [arXiv:astro-ph/9702166].

[9] A. Lewis, J. Weller and R. Battye, Mon. Not. Roy. Astron. Soc. 373 (2006) 561 [arXiv:astro-ph/0606552].

[10] A. Lewis, A. Challinor and A. Lasenby, Astrophys. J. 538, 473 (2000) [arXiv:astro-ph/9911177].

[11] A. Lewis and S. Bridle, Phys. Rev. D 66, 103511 (2002) [arXiv:astro-ph/0205436].

[12] W. C. Jones et al., Astrophys. J. 647, 823 (2006); F. Piacentini et al., Astrophys. J. 647, 813 (2006), Astrophys. J. 647, 833 (2006).

[13] M. L. Brown et al. [QUaD collaboration], Astrophys. J. 705, 978 (2009) [arXiv:0906.1003 [astro-ph.CO]].

[14] C. L. Reichardt et al., Astrophys. J. 694, 1200 (2009) [arXiv:0801.1491 [astro-ph]].

[15] H. C. Chiang et al., Astrophys. J. 711, 1123 (2010) [arXiv:0906.1181 [astro-ph.CO]].

[16] D. H. Lyth and A. Riotto, Phys. Rept. 314, 1 (1999). [arXiv:hep-ph/9807278].

[17] [Planck Collaboration], [arXiv:astro-ph/0604069].

[18] W. H. Kinney, Phys. Rev. D 66, 083508 (2002) [arXiv:astro-ph/0206032].

[19] R. Easther and W. H. Kinney, Phys. Rev. D 67, 043511 (2003) [arXiv:astro-ph/0210345].

[20] W. H. Kinney, E. W. Kolb, A. Melchiorri and A. Riotto, Phys. Rev. D 69, 103516 (2004) [arXiv:hep-ph/0305130]. 